EXTENDED REPORT

\title{
Autoimmune manifestations in human myelodysplasia: a positive correlation with interferon regulatory factor-1 (IRF-1) expression
}

\author{
S Giannouli, D Tzoanopoulos, K Ritis, G Kartalis, H M Moutsopoulos, M Voulgarelis
}

Ann Rheum Dis 2004;63:578-582. doi: 10.1136/ard.2003.012948

See end of article for authors' affiliations

.....................

Correspondence to: Dr Haralampos M Moutsopoulos, Department of Pathophysiology, Medical School, National

University of Athens, $75 \mathrm{M}$

Asias St, Goudi, 11527

Athens, Greece

hmoutsop@med.voa.gr

Accepted 21 July 2003
Background: Patients with myelodysplasia may have autoimmune manifestations (AIM). Interferon regulatory factor-1 (IRF-1) is a transcription factor involved in interferon signalling, leukaemogenesis, and the development of the immune system.

Objectives: To determine whether IRF-1 is implicated in the pathophysiology of AIM in myelodysplasia. Methods: 14 patients with myelodysplasia were studied, seven with AIM and seven without. Five patients with vasculitis and seven normal subjects served as controls. The expression of IRF-1 was studied in bone marrow mononuclear cells taken from patients and controls, using a relative quantitative reverse transcriptase polymerase chain reaction.

Results: A 10-fold reduction in full length IRF-1 mRNA was detected in the myelodysplasia patients without AIM compared with the normal controls. In contrast, the group with AIM had increased IRF-1 transcripts, to a level almost equal to that observed in patients with vasculitis and normal controls.

Conclusions: Myelodysplasia patients without IRF-1 expression had a decreased incidence of AIM. Thus the absence of IRF-1 transcription factor appears to protect against the development of autoimmunity in myelodysplasia.
$P$

rimary myelodysplastic syndromes are clonal disorders of the haematopoietic stem cell characterised by ineffective and dysplastic haematopoiesis and peripheral cytopenias. A distinct subset of patients with this disease have overt autoimmune manifestations (AIM), the underlying pathogenesis and prognostic significance of which remain controversial. ${ }^{1}$ Interferon regulatory factor-1 (IRF-1) has been identified as a transcriptional activator of the interferon- $\beta$ (IFN $\beta$ ) as well as of IFN inducible genes. ${ }^{2}$ IRF- 1 is involved in DNA damage induced apoptosis and cell cycle arrest, and suppresses tumorigenicity of transformed cells, playing an essential role in cell growth control and surveillance against malignant development. ${ }^{3}$ Deletions and somatic mutations of the IRF-1 gene have been implicated in the multiphase process leading to human myelodysplasia and leukaemic evolution. ${ }^{4}$ Furthermore, alternative splicing of the IRF-1 mRNA resulting in truncated non-functional transcripts has been observed at high frequency in patients with myelodysplasia. ${ }^{5}$ Thus accelerated exon skipping of IRF-1 has been considered a possible mechanism of functional inactivation of IRF-1 in myelodysplasia.

Apart from its tumour suppressor function, IRF-1 regulates multiple stages of the immune response such as induction of IL-12 and Thl differentiation, as well as major histocompatibility complex (MHC) II, CD8 T, and natural killer (NK) cell maturation. ${ }^{67}$ IRF-1 is involved in the induction of the inflammatory response, regulating the expression of inflammation related enzymes such as inducible nitric oxide synthase and cyclo-oxygenase-2, and amplifying tumour necrosis factor (TNF) induced vascular cell adhesion molecule 1 expression. ${ }^{8}$ This evidence shows that IRF-l is a transcriptional activator linking immune response and oncogenesis.

Our aim in this study was to investigate IRF-1 mRNA expression in bone marrow mononuclear cells from patients with myelodysplasia, with or without AIM, to identify its possible role in immune deregulation in preleukaemic states.

\section{METHODS}

\section{Patients}

Seven myelodysplastic patients with AIM (group A) diagnosed in our department between 1999 and 2002 were evaluated. Seven other myelodysplastic patients without AIM (group B), randomly selected from a pool of 75 patients followed up in our department, were also included in the study. Five patients with systemic vasculitis (group C; one with polyarteritis nodosa and four with Wegener's granulomatosis) and seven normal individuals (group D) served as controls. The selection of the patients with systemic vasculitis as controls was based on the fact that vasculitic syndromes were the commonest type of AIM in group A patients.

Medical history, date of diagnosis, and date of presentation of AIM, if any, were recorded. Clinical examination, haematological and biochemical variables, serum electrophoresis, and autoantibody profile were monitored. Bone marrow aspirates, biopsies, and cytogenetic data from all patients and controls were obtained. There was no evidence of infection at the time of bone marrow aspiration. Neither chemotherapy nor immunosuppression had been given before the bone marrow collection. Dysplastic changes and dyspoiesis in bone marrow aspirates were defined according to the criteria of Bennett.' Myelodysplastic patients were classified according to the French/American/British (FAB) classification, ${ }^{9}$ and were also categorised as low, intermediate, or high risk myelodysplasia using the international prognostic scoring system (IPSS). ${ }^{10}$ Karyotypic analysis of all samples by means of GTG banding was undertaken. Samples were obtained with the patient's written informed consent.

Abbreviations: AIM, autoimmune manifestations; $\mathrm{BMI}$, band mean intensity; IRF-1, interferon regulatory factor-1 


\section{Expression of IRF-1 full length mRNA (IRF-1-FL)}

After bone marrow mononuclear cell isolation by gradient centrifugation and subsequent total RNA extraction by TRIzol (Gibco-BRL/Life Technologies), a relative quantitative reverse transcriptase polymerase chain reaction (RT-PCR) was done, using the QuantumRNA classic II $18 \mathrm{~S}$ kit (Ambion), as previously described. ${ }^{11}$ Relative quantitation compares IRF-1 transcript between multiple samples, using 18S rRNA as a coamplified internal control for sample normalisation (dual quantitative relative RT-PCR). Results are expressed as the ratio between the optical density of IRF1 amplicon and the optical density of the $18 \mathrm{~S}$ amplicon. This yields a corrected relative value for the IRF-1 product in each sample.

In order to ensure proper semiquantitative RT-PCR, the reaction is terminated when the products from both the internal control and the IRF-1 genes are within their exponential phase. Because 18S rRNA is a gene of high abundance and its amplification surpasses the exponential phase with very few PCR cycles, we used competimers to reduce the amplification signal. The $18 \mathrm{~S}$ rRNA competimers are identical to 18S rRNA primers, but are modified at their 3' end to block extension of DNA polymerase. Mixing 18S rRNA primers with increasing amounts of 18S RNA competimer allows a reduction in amplification efficiency of the $18 \mathrm{~S}$ rRNA template without loss of relative quantitation. Thus relative RT-PCR provides an estimate of the relative changes in IRF-1FL expression between samples.

Briefly, a nested PCR assay was used to amplify the entire IRF-1 mRNA. PCR was carried out using the outer primers IRF-IU: 5'GGCGGCCTTAAGAACCAG3' and IRF-IL: 5'ACTTGGCAGTGGGGTCACA 3', and the inner primers 67IRF-1: 5'TTAAGAACCAGGCAACC3' and 1055IRF-1: 5'TGTAGACTCAGCCCAATA3', according to the cDNA sequences of IRF-1 reported by Maruyama et al, ${ }^{12}$ providing a product of 1006 base pairs (bp). 18S rRNA was used as an internal control in the nested PCR, after cycling parameters, linear range, and $18 \mathrm{~S}$ primer/competimer ratio were optimised according to the manufacturer's instructions. The resulting PCR products were separated on a $2 \%$ agarose gel. Gels were photographed using a Kodak DC40 camera and the band mean intensity (BMI) of full length IRF-1 and 18S was analysed using Kodak DS, ID image analysis software, v 3.0.1. (Eastman Kodak).

\section{Sequencing}

The MWG Biotech AG sequencing service (http:// www.mwgdna.com) was used for sequence analysis of smaller size PCR products obtained after amplification of IRF-1-FL, corresponding to aberrantly spliced IRF-1 mRNAs.

\section{Statistical analysis}

The difference in the BMI of IRF-1-FL between the four groups was investigated using the Kruskal-Wallis test. Individual comparisons between two groups were made using the Mann-Whitney test with Bonferroni's correction.

\section{RESULTS}

\section{Patient characteristics}

Demographic and clinical characteristics of group A and B patients, subdivided according to $\mathrm{FAB}$ classification and the IPSS system, together with haematological and cytogenetic data are given in table 1. Previous exposure to agents predisposing to myelodysplasia was not reported. In group A, five patients were of high risk, one was of intermediate risk, and one of low risk according to the IPPS for myelodysplasia. ${ }^{10}$ Five patients were of high risk in group $\mathrm{B}$.

\section{AIM in myelodysplasia patients}

The AIM detected in group A are shown in table 2. The majority of our patients had vasculitic overlap syndromes, leucocytoclastic vasculitis being the commonest manifestation. ${ }^{13}$ Needle biopsy of the lung in case 1 showed small vessel vasculitis with polymorphonuclear infiltrates around inflamed vessels. A deep, open surgical biopsy, including skin, subcutaneous tissue, and muscle, revealed oedema and infiltrations of polymorphonuclear neutrophils involving the media and the intima of small arteries in case 7. Biopsy of the sural nerve in the same patient showed widespread inflammation of small arteries of the epinevrium, with a mixture of axonal loss and segmental demyelination. There was no evidence of autoimmune disease prior to the diagnosis of myelodysplasia. No correlation between FAB classification and IPSS grade with predisposition to development of AIM was observed.

\section{Dissimilar expression of IRF-1 in myelodysplasia patients with and without AIM}

Low expression of IRF-1-FL was observed in group B patients. After comparative normalisation of every sample according to 18S RNA expression, the mean (SD) BMI of IRFl-FL in the seven normal individuals (group D) was 10248 (3312) pixels, while in group B it was 872 (830) pixels, a 10fold reduction $(p=0.007)$. Importantly, four of the seven patients in group B showed undetectable levels of IRF-1-FL. The low expression of IRF-1-FL in these patients was associated with the presence of aberrant spliced mRNAs, resulting from skipping of the exons 2, 6, 7, 8, and 9 in patient 4, and from skipping of exon 2 in patient 1 . In contrast, expression of IRF-1-FL was observed in all group A patients. The full length BMI in this group was 5895 (1858) pixels, only marginally different from the normal controls (group D; $p=0.051$ ), and not significantly different from group C patients with systemic vasculitis (5834 (2539) pixels; $\mathrm{p}=0.627)$. Normal controls, patients with systemic vasculitis, and myelodysplastic patients with AIM all expressed the IRF-1-FL. Skipping of exons 2 or 2 and 3 and other spliced mRNAs in these groups was detected after extended PCR amplification (more than 28 cycles of nested PCR), while under the quantification conditions described here, these deletions were not established. IRF-1 mRNA expression pattern and its relative expression to 18s rRNA in the four study groups are shown in figs 1 and 2, respectively.

\section{DISCUSSION}

In this study we examined the expression pattern of IRF-1 in 14 patients with myelodysplasia (seven without AIMS and seven with AIM). In order to achieve accuracy in the measurement of IRF-1-FL and to characterise possible truncated transcripts we used a nested RT-PCR which amplified the entire IRF-1 gene. We have used this successfully to study IRF-1 expression in patients with chronic myeloid leukaemia. ${ }^{11}$ Five patients with systemic vasculitis and seven normal individuals were also studied and used as controls in order to make our results more reliable. Amplification of IRF-1-FL showed the presence of alternatively spliced mRNAs and a parallel reduction in IRF-1-FL in all seven myelodysplastic patients without AIM; in contrast, all seven with coexisting AIM expressed IRF-1-FL at a level almost equal to that of patients with systemic vasculitis and normal controls. In our study, healthy controls express the highest levels of IRF-1 mRNA, while patients with vasculitis express less. Our data on IRF-1 expression in controls seem to be contradictory to the hypothesis that IRF-1 plays a role in inflammation.

IRF-1 protein is constantly produced by all cell types examined and has been found to be induced by several 
Table 1 Demographic, haematological, and cytogenetic data

\begin{tabular}{|c|c|c|c|c|c|c|c|}
\hline Cases & Age/sex & FAB & IPSS & Cytogenetics & $\mathrm{Hg}(\mathrm{g} / \mathrm{dl})$ & $\begin{array}{l}\text { WBC }\left(\times 10^{3} /\right. \\
\left.\mathrm{mm}^{3}\right)\end{array}$ & PLT $\left(\times 10^{3} / \mathrm{mm}^{3}\right)$ \\
\hline \multicolumn{8}{|c|}{ Group A } \\
\hline 1 & $67 / M$ & RAEB & $\mathrm{H}$ & $\begin{array}{l}46 X Y \\
46 X X \text {, del } 5 q \text {, }\end{array}$ & 9.2 & 1.8 & 17 \\
\hline 2 & $56 / \mathrm{F}$ & RAEB-T & $\mathrm{H}$ & $t(2 ; 11)$ & 9.7 & 3 & 80 \\
\hline 3 & $74 / F$ & CMML & $\mathrm{H}$ & $\begin{array}{l}46 \mathrm{XX} \\
44 \mathrm{XY},-5,-7, \mathrm{del}\end{array}$ & 9.9 & 7.3 & 65 \\
\hline 4 & $80 / M$ & CMML & $\mathrm{H}$ & $3 q$ & 9.3 & 13.7 & 90 \\
\hline 5 & $51 / \mathrm{F}$ & RA & $\mathrm{L}$ & $46 X X$ & 10.5 & 2.9 & 104 \\
\hline 6 & $67 / M$ & RARS & 1 & $47 X Y+8$ & 9.4 & 4.8 & 100 \\
\hline 7 & $59 / \mathrm{M}$ & RAEB-T & $\mathrm{H}$ & $46 X Y$, del $20 q$ & 7.5 & 1.5 & 15 \\
\hline \multicolumn{8}{|c|}{ Group B } \\
\hline 1 & $75 / M$ & RAEB-T & $\mathrm{H}$ & $47 X Y,+11$ & 9 & 1 & 100 \\
\hline 2 & $63 / M$ & RAEB-T & $\mathrm{H}$ & $47 X Y,+8$ & 8 & 1 & 25 \\
\hline 3 & $79 / M$ & RA & $\mathrm{H}$ & $46 X Y$ & 9 & 1 & 2 \\
\hline 4 & $78 / M$ & RARS & $\mathrm{H}$ & $46 X Y$ & 9.4 & 4.2 & 239 \\
\hline 5 & $75 / M$ & CMML & $\mathrm{H}$ & $46 X Y$ & 9.7 & 21 & 1 \\
\hline 6 & $68 / \mathrm{F}$ & RA & $\mathrm{L}$ & $46 \mathrm{XX}$, del $5 q$ & 9 & 5.1 & 250 \\
\hline 7 & $65 / M$ & RARS & $\mathrm{L}$ & $46 X Y$ & 10.1 & 4.9 & 210 \\
\hline
\end{tabular}

CMML, chronic myelomonocytic leukaemia; $F$, female; $F A B$, French/American/British classification; $\mathrm{H}$, high; $\mathrm{Hb}$, haemoglobin; I, intermediate; IPSS, international prognostic score system; L, low; M, male; PLT, platelet count; RA, refractory anaemia; RAEB, refractory anaemia with excess blasts; RAEB-T, refractory anaemia with excess blasts in transformation; RARS, refractory anaemia with ring sideroblasts; WBC, white blood cell count.

stimuli such as hormones, cytokines, interferons, and viral infections, with consequent variability in its expression levels. Moreover, IRF-1 mRNA expression depends on the activation and differentiation status of each cell, as well as being cell cycle regulated. In addition, the IRF-1 mRNA has a short half life and this permits rapid changes of its level. Consequently, the interpretation of quantitative differences of IRF-1 expression in normal individuals remains uncertain. ${ }^{8}$ On the other hand, the effect of impaired IRF-1 expression and function on tumorigenesis and myelodysplasia is not in doubt, and the immunomodulatory function of IRF-1 has been identified experimentally. It is not logical that the complex regulation of IRF-1 mRNA expression should affect controls differently from patients with myelodysplasia, but the fact that the expression is dissimilar between myelodysplastic patients with and without AIM appears interesting. These results must be confirmed by functional assays.

Table 2 Autoimmune/inflammatory manifestations in group A patients

\begin{tabular}{|c|c|c|}
\hline Case & Clinical manifestations & Serological data \\
\hline 1 & $\begin{array}{l}\text { Relapsing polychondritis, } \\
\text { leucocytoclastic vasculitis, non- } \\
\text { infectious pulmonary infiltrates }\end{array}$ & Hypergammaglobulinaemia \\
\hline 2 & $\begin{array}{l}\text { Primary biliary cirrhosis, thyroiditis } \\
\text { Hashimoto's, leucocytoclastic } \\
\text { vasculitis }\end{array}$ & $\begin{array}{l}\text { ANA:1/160, AMA:1/640, } \\
\text { positive DAT, } \\
\text { hypergammaglobulinaemia }\end{array}$ \\
\hline 3 & $\begin{array}{l}\text { Leucocytoclastic vasculitis } \\
\text { Hypergammaglobulinaemia, RF:1/ } \\
60\end{array}$ & \\
\hline 4 & Leucocytoclastic vasculitis & Hypergammaglobulinaemia \\
\hline 5 & $\begin{array}{l}\text { Symmetric polyarticular synovitis, } \\
\text { optic neuritis, erythema nodosum }\end{array}$ & $\begin{array}{l}\text { ANA: } 1 / 160 \text {, } \\
\text { hypogammaglobulinaemia }\end{array}$ \\
\hline 6 & $\begin{array}{l}\text { Polymyalgia rheumatica/temporal } \\
\text { arteritis, leucocytoclastic vasculitis } \\
\text { Hypergammaglobulinaemia, RF:1/ } \\
60\end{array}$ & \\
\hline 7 & $\begin{array}{l}\text { Microscopic polyangiitis, livedo } \\
\text { reticularis, myositis, peripheral } \\
\text { neuropathy, leucocytoclastic vasculitis } \\
\text { Hypergammaglobulinaemia, positive } \\
\text { DAT }\end{array}$ & \\
\hline
\end{tabular}

AMA, antimitochondrial antibodies; ANA, antinuclear antibodies; DAT, direct antiglobulin test; RF, rheumatoid factor.
Recent studies in AML/myelodysplasia showed low expression of the IRF-1 gene as a result of accelerated exon skipping, leading to tumour suppressor gene inactivation. ${ }^{5}$ In our study, low expression of IRF-1-FL was established in all seven myelodysplasia patients without AIM; this was in conjunction with exon skipping events, further supporting the possible role of IRF-1 gene inactivation in the pathology and progression of the disease. Surprisingly, the expression pattern of IRF-1 mRNA was completely distinct in myelodysplasia patients with AIM, revealing intact IRF-1-FL expression and the consistent absence of exon skipping in all seven cases examined. Furthermore, IRF-1-FL expression in these patients was almost equal to that observed in patients with systemic vasculitis. Multiple alterations have been implicated in the pathogenesis of myelodysplasia, such as oncogene activation (Ha-ras, c-fos), cell cycle inhibitor deregulation (p21), and inactivation of tumour suppressor genes (p53, IRF-1). These alterations may affect haematopoietic stem cells, providing them with a growth advantage and accelerating the progression to leukaemic evolution, but the initial damaging genetic event has not yet been identified. Indeed the heterogeneity of the clinical manifestations and prognosis among the different categories of myelodysplasia parallels the complexity of their pathogenic events. In these complex genetic alterations, the deregulation of IRF-1 gene by accelerated exon skipping seems to be a critical event in myelodysplasia. However, the finding of IRF-1-FL expression in our group A (myelodysplastic patients with AIM) argues against a causative role of exon skipping mediated IRF-1 inactivation in this specific group of patients, and indicates another underlying mechanism of leukaemic transformation. As FAB subclasses and IPSS categories were spread almost equally over the two distinct subgroups of myelodysplastic patients, the dissimilar expression could not be related to anything other than the autoimmune profile of these patients.

The estimated incidence of AIM in myelodysplasia was as high as $10 \%$ in previous studies. ${ }^{1}$ Despite speculation that immunological mechanisms are involved in this phenomenon, the exact pathophysiology remains unknown. A decrease in circulating NK cell number accompanied by an absolute lymphopenia and functional abnormalities of macrophages, CD4 lymphocytes, and NK cells, with deficient production of interferon a, have also been reported. Impaired 

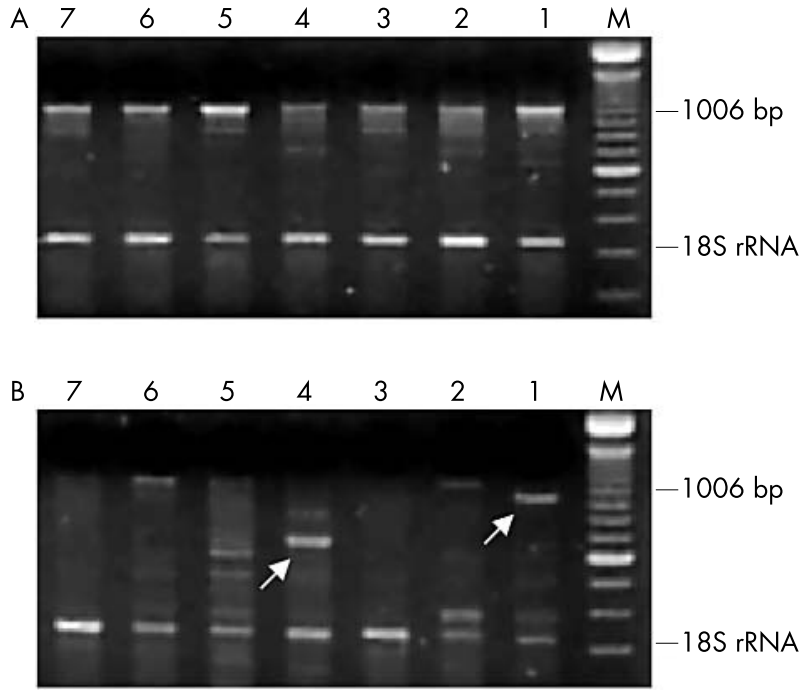

C
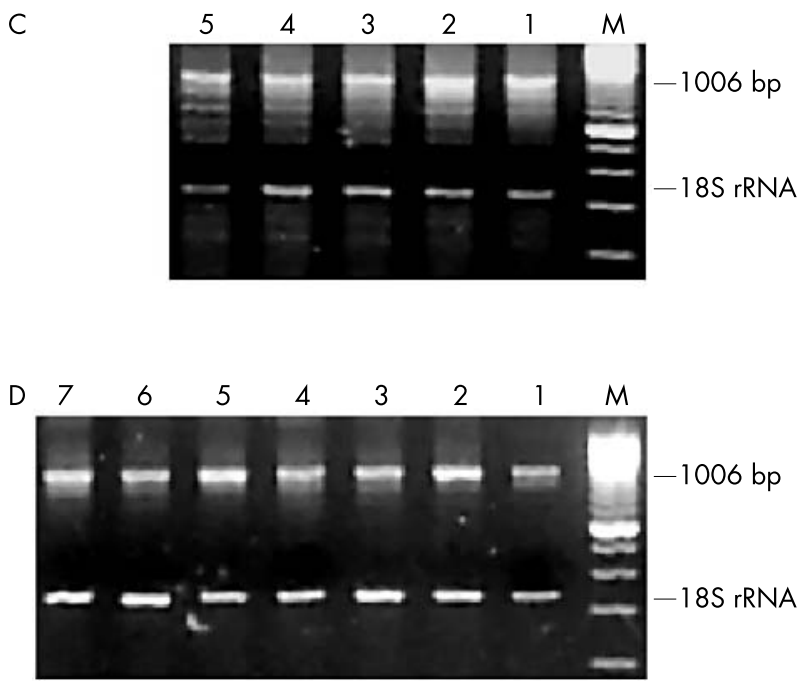

Figure 1 Reverse transcriptase polymerase chain reaction (RT-PCR) amplifications representing interferon regulatory factor-1 (IRF-1) mRNA and $18 \mathrm{~S}$ rRNA in patients and controls. (A) Group A, lanes 1-7: seven myelodysplasia patients with autoimmune manifestations. (B) Group B, lanes 1-7: seven myelodysplasia patients without autoimmune manifestations. (C) Group C, lanes 1-5: five patients with systemic vasculitis. (D) Group D, lanes 1-7: seven normal individuals. Myelodysplasia patients from group B have decreased levels of full length IRF-1 mRNA expression in association with exon skipped products, as in case 1 and 4 (arrows). On the other hand, myelodysplasia patients from group A express the full length of IRF-1 mRNA (a 1006 bp product), without exon skipped products. The control groups ( $C$ and $D)$ also show expression of the full length of IRF-1 mRNA. M, 100 bp marker (Gibco).

B cell function is indicated by hypergammaglobulinaemia, monoclonal paraproteinaemia, and the presence of tissue specific and non-tissue-specific autoantibodies. ${ }^{1}$ It has been hypothesised that these aberrations influence immunoregulatory $\mathrm{T}$ and $\mathrm{B}$ cell interactions predisposing to the development of autoimmune inflammatory disorders in preleukaemic states. In this regard, overactive antigenic presentation resulting from disordered monocyte/macrophage function leads to persistent immune stimulation and upregulated cytokine secretion, allowing the activation of inflammatory mediators.

IRF-1 was originally discovered as a regulator of the innate immune system, being related to $\mathrm{T}$ cell maturation in the

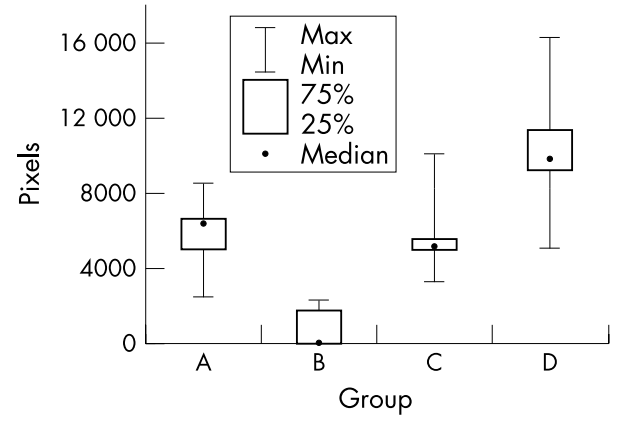

Figure 2 Box plots showing the median, minimum, maximum, and quartile pixel values for each group of patients. The relative interferon regulatory factor-1 (IRF-1) mRNA expression to 18s rRNA levels is presented as box plots with median and interquartile ranges for each group. Group A, seven myelodysplastic patients with autoimmune manifestations; group B, seven myelodysplastic patients without autoimmune manifestations; group $C$, five patients with systemic vasculitis; group $D$, seven normal individuals.

thymic environment and regulating the expression of the IL15 gene that is required for survival and proliferation of NK cells in vivo. ${ }^{7}$ Recent evidence that IRF-l promotes Thl differentiation through the production of IL-12 by macrophages further supports the hypothesis that IRF-1 may link innate and adoptive immunity. ${ }^{6}$ Furthermore IRF-1 is involved in the induction of the inflammatory response regulating the expression of inflammation related enzymes, such as inducible nitric oxide synthase and cyclo-oxygenase2 , and amplifying TNF induced vascular cell adhesion molecule 1 expression. ${ }^{8}$ These studies led us to hypothesise that IRF-1 is involved in the pathogenesis of AIM in myelodysplasia. Indeed, myelodysplastic patients with AIM possessed IRF-1 mRNA levels equal to those observed in patients with vasculitis without myelodysplasia, while those without AIM were characterised by suppression of IRF-1 mRNA expression. Our results are in agreement with in vivo models of IRF-1 deficient mice, showing a reduced incidence and severity of type II collagen induced arthritis and allergic encephalomyelitis. ${ }^{14}$ These studies on IRF-1 deficient mice have revealed that mice lacking IRF-l have a decreased incidence and severity of type II collagen induced arthritis and experimental allergic encephalomyelitis compared with wild type mice.

In these studies the investigators not only implied a protective role of the absence of IRF-1, but they also concluded that IRF-1 plays a part in promoting inflammation and autoimmunity. They proposed that the inhibition of this transcription factor may be a novel form of treatment for controlling inflammatory diseases. Despite the fact that these experimental data cannot been applied to humans, our results point in that direction. The two groups of myelodysplastic patients that we studied-with and without autoimmune manifestations-form an ideal in vivo model, comparable with the animal model mentioned above.

Our study suggests that IRF-1 offers a unique opportunity to study the immunoregulatory changes in preleukaemic states. While our results show an association of increased IRF-1 mRNA expression with autoimmune manifestations in myelodysplasia, they do not yet allow us to conclude that IRF-1 is definitely involved in the pathogenesis of autoimmunity, as no functional experiments were done. Further investigation of IRF-l expression in systemic autoimmune disorders could be intriguing.

\section{ACKNOWLEDGEMENTS}

We wish to thank Dr A G Tzioufas, M N Manoussakis, and E Zintzaras for their support and encouragement. The study was 
supported by research funding from the General Secretariat of Research and Development and from Boehringer-Ingelheim Hellas Co.

\section{Authors' affiliations}

S Giannouli, H M Moutsopoulos, M Voulgarelis, Department of Pathophysiology, Medical School, National University of Athens, Greece D Tzoanopoulos, K Ritis, G Kartalis, First Division of Internal Medicine, Democritus University of Thrace, Alexandroupolis, Greece

\section{REFERENCES}

1 Hamblin TJ. Immunological abnormalities in myelodysplastic syndromes. Semin Hematol 1996:33:150-62.

2 Miyamoto M, Fujita T, Kimura Y, Maruyama M, Harada H, Sudo Y, et al. Regulated expression of a gene encoding a nuclear factor, IRF-1, that specifically binds to IFN-beta gene regulatory elements. Cell 1988;54:903-13.

3 Tanaka N, Ishihara M, Kitagawa M, Harada H, Kimura T, Matsuyama T, et al. Cellular commitment to oncogene-induced transformation or apoptosis is dependent on the transcription factor IRF-1. Cell 1994;77:829-39.

4 Willman CL, Sever CE, Pallavicini MG, Harada H, Tanaka N, Slovak ML, et al. Deletion of IRF-1, mapping to chromosome $5 \mathrm{q} 31.1$, in human leukemia and preleukemic myelodysplasia. Science 1993;259:968-71.

5 Harada H, Kondo T, Ogawa S, Tamura T, Kitagawa M, Tanaka N, et al. Accelerated exon skipping of IRF-1 mRNA in human myelodysplasia/ leukemia; a possible mechanism of tumor suppressor inactivation. Oncogene 1994;9:3313-20.

6 Salkowski CA, Kopydlowski K, Blanco J, Cody MJ, McNally R, Vogel SN. IL12 is dysregulated in macrophages from IRF-1 and IRF-2 knockout mice. $\mathrm{J}$ Immunol 1999;163:1529-36.

7 Ogasawara K, Hida S, Azimi N, Tagaya Y, Sato T, Yokochi-Fukuda T, et al. Requirement for IRF-1 in the microenvironment supporting development of natural killer cells. Nature 1998;391:700-3.

8 Kroger A, Koster M, Schroeder K, Hauser H, Mueller PP. Activities of IRF-1. $J$ Interferon Cytokine Res 2002;22:5-14.

9 Bennett JM, Catovsky D, Daniel MT, Flandrin G, Galton DA, Gralnick HR, et al. Proposals for the classification of the myelodysplastic syndromes. Br J Haematol 1982:51:189-99.

10 Greenberg P, Cox C, LeBeau MM, Fenaux P, Morel P, Sanz G, et al. International scoring system for evaluating prognosis in myelodysplastic syndromes. Blood 1997;89:2079-88.

11 Tzoanopoulos D, Speletas M, Arvanitidis K, Veiopoulou C, Kyriaki S Thyphronitis $G$, et al. Low expression of interferon regulatory factor-1 and identification of novel exons skipping in patients with chronic myeloid leukaemia. Br J Haematol 2002; 1 19:46-53.

12 Maruyama M, Fujita T, Taniguchi T. Sequence of a cDNA coding for human IRF-1. Nucleic Acids Res 1989;17:3292.

13 Bloch DA, Michel BA, Hunder GG, McShane DJ, Arend WP, Calabrese LH, et al. The American college of Rheumatology 1990 criteria for the classification of vasculitis. Patients and methods. Arthritis Rheum 1990;33:1068-73.

14 Tada Y, Ho A, Matsuyama T, Mak TW. Reduced incidence and severity of antigen-induced autoimmune diseases in mice lacking interferon regulatory factor-1. J Exp Med 1997; 185:231-8. 